Disponível em:

http://editora.unoesc.edu.br/index.php/race

RACE, Joaçaba, v. 16, n. 3, p. 933-956, set./dez. 2017

\title{
A EFETIVIDADE DA GOVERNANÇA CORPORATIVA SOBRE O RISCO DOS ATIVOS DA BM\&FBOVESPA ${ }^{1}$
}

The effectiveness of corporate governance on the risk of BM\&FBovespa's assets

Cássio Nóbrega Besarria

E-mail: cassiodanobrega@yahoo.com.br

Doutor em Economia pela Universidade Federal de Pernambuco; Mestre em Economia pela Universidade Federal da Paraíba; Professor na Universidade Federal da Paraíba. Endereço para contato: Cidade Universitária, Castelo Branco III, 58051-085, João

Pessoa, Paraíba, Brasil.

Herickson Santos Silva

E-mail: hss.h94@gmail.com

Graduado em Economia pela Universidade Federal da Paraíba.

Artigo recebido em 14 de abril de 2017. Aceito em 24 de julho de 2017.

\footnotetext{
${ }^{1}$ Agradecemos os pertinentes comentários e sugestões dos pareceristas anônimos. Erros ainda existentes são de responsabilidade dos autores.
} 
Resumo

Com este artigo teve-se o propósito de verificar se a utilização dos métodos de governança corporativa adotados pelas empresas listadas na Bolsa de Valores, Mercadorias e Futuros de São Paulo (BM\&FBovespa) diminui o risco nos ativos. Utilizando cotações do período de 01 de junho de 2012 a 04 de julho de 2016, este estudo se divide em três etapas: no primeiro momento, as cotações são otimizadas e analisadas quanto ao desempenho das três carteiras de variância mínima referentes aos diferentes níveis de governança corporativa: Nível 1 (N1), Nível 2 (N2) e Novo Mercado (NM). Na segunda etapa é estimado por MQO o modelo de precificação de ativos de Sharpe-Lintner-Black, o Capital Asset Pricing Model (CAPM). Na terceira etapa são comparados os desempenhos de carteiras representativas para as ações classificadas em diferentes níveis de governança com índices de governança propostos pela BM\&FBovespa. De acordo com os resultados obtidos, foi observado que os ativos que possuem maior nível de transparência tendem a apresentar menores níveis de variância ou risco.

Palavras-chave: Governança corporativa. Mercado financeiro. Risco financeiro.

\section{Abstract}

The purpose of this article was to verify if the use of the corporate governance methods adopted by the BM\&FBovespa listed companies reduces the risk in the assets. Using prices from June 1, 2012 to July 4, 2016, this study is divided into three stages: at the first moment, the three minimum variance portfolios referring to the different corporate governance levels are optimized and analyzed: 1 (N1), Level 2 (N2) and Novo Mercado (NM). In the second stage, the asset pricing model of Sharpe-Lintner-Black, the Capital Asset Pricing Model (CAPM), is estimated by MQO. In the third step, the performance of representative portfolios is compared to the shares rated at different levels of governance with governance indexes proposed by $B M$ \& FBovespa. According to the results obtained, it is observed that the assets with the highest level of transparency tend to have lower levels of variance or risk.

Keywords: Corporate governance. Financial market. Financial risk.

\section{INTRODUÇÃO}

De acordo com Sá (1999) e Carmona (2009), o mercado acionário é uma forma alternativa de captação de recursos para as empresas que buscam financiamentos de longo prazo, ampliando a capacidade de realizar investimentos. O mercado de ações tem como principal finalidade transferir poupança de agentes superavitários para empresários que pretendem realizar investimentos com custo inferior ao sistema bancário, e o seu desenvolvimento está diretamente ligado ao nível e à transparência 
das informações, previsibilidade econômica, sistema tributário, eficiência jurídica, entre outros fatores.

O mercado de ações no Brasil passou a ter maior relevância após a adoção do plano real, uma vez que este trouxe maior estabilidade econômica e contribuiu para a redução das incertezas em relação à economia brasileira. Aliados a esses componentes, outros mecanismos têm contribuído para o desenvolvimento desse mercado, como mudanças regulatórias e adoção dos princípios e práticas da boa governança corporativa com a criação do Instituto Brasileiro de Conselheiros de Administração (IBCA), que, posteriormente, ficou conhecido como Instituto Brasileiro de Governança Corporativa (IBGC).

Tomando como base os componentes apresentados anteriormente, neste artigo tem-se o propósito de analisar os efeitos do grau de transparência das empresas, representado pelos diferentes níveis de governança corporativa, sobre a relação risco versus retorno dos ativos. Lançados no fim do ano 2000 pela Bolsa de Valores de São Paulo (Bovespa), os segmentos de empresas Novo Mercado (NM) e os Níveis Diferenciados de Governança Corporativa (NDGC) introduziram um novo objetivo para as empresas brasileiras: alcançar maior grau de credibilidade e segurança com transparência.

Esses diferentes segmentos indicam o grau de gestão transparente praticado e alcançado pela empresa, conforme legislação vigente aplicada pela BM\&FBovespa. Nesse caso, o segmento de Novo Mercado representa a lista das empresas que apresentam o melhor nível de governança e o índice NDGC, que é subdividido em duas categorias: Nível 1 e Nível 2, podendo classificá-los com menor e maior grau de transparência, respectivamente.

Alguns estudos aplicados à economia brasileira já procuraram captar esses efeitos. Um desses estudos foi o proposto por Silveira (2004), que encontrou indícios de que o grau de governança corporativa influencia significativamente o grau de alavancagem financeira das firmas, demonstrando uma das estratégias eficientes de atração de indivíduos que buscam realizar investimentos com menor risco.

Posteriormente, Monte et al. (2010) analisaram a composição risco e persistência da volatilidade de três carteiras de variância mínima formadas, respectivamente, por ativos de empresas que fazem parte dos níveis de governança Nível 1 (N1), Nível 2 (N2) e Novo Mercado (NM). Os resultados encontrados mostraram que carteiras formadas por ações com maior grau de transparência (NM) são menos arriscadas que as compostas por ativos das empresas dos Níveis 1 e 2 . 
Além desses, Besarria et al. (2015) verificaram se a qualidade das informações prestadas pelas empresas e a ampliação dos direitos societários reduzem os riscos de investimento. Para tanto, utilizou-se um arcabouço teórico baseado nos desdobramentos do CAPM condicional e, empiricamente, analisou-se a validade das estimativas da volatilidade condicional utilizando os três segmentos de governança corporativa do N1, N2 e NM. Os autores mostraram que o intervalo de variação dos betas da carteira de Novo Mercado é inferior aos das demais carteiras.

Além disso, Besarria et al. (2015) mostraram que as ações das empresas listadas no Novo Mercado apresentaram coeficiente de persistência inferior aos obtidos pelas carteiras N1 e N2, indicando que os efeitos dos choques negativos na volatilidade dos retornos levam menos tempo para se dissipar nessa carteira. E, por meio das estimativas das volatilidades, também foi possível comprovar que as ações das empresas do Novo Mercado são as menos voláteis, seguidas pelas ações classificadas no Nível 2 e no Nível 1 (mais voláteis), respectivamente. Esses resultados reforçam a hipótese de que a qualidade das informações prestadas pelas empresas e a ampliação dos direitos societários reduzem os riscos de investimento.

De forma complementar, Silva e Câmara (2015) obtiveram resultados que comprovam a hipótese de que a valorização das empresas é influenciada diretamente pelo nível de governança, mostrando que quanto maior a transparência, maior a confiança gerada nos investidores, influenciando a procura e a valorização dos ativos.

Com isso, neste estudo pretendeu-se investigar se as práticas de governança implicam a redução do risco dos investimentos. Esta análise é tratada em três partes: inicialmente, são formadas e analisadas três carteiras, cada uma representando um dos níveis de transparência já descritos, sendo utilizado o processo de análise e seleção de carteiras proposto por Markowitz (1952). Na segunda parte, as carteiras de variância mínima são utilizadas para estimar o Modelo de Precificação de Ativos de Capital $(C A P M)$, que avalia a suscetibilidade dos ativos às variações do mercado.

A terceira etapa tem o propósito de comparar o desempenho das carteiras de mercado propostas pela BM\&FBovespa, utilizadas para representar as corporações classificadas com diferentes níveis de governança corporativa, com carteiras de governança que foram criadas a partir do princípio da minimização de risco proposto por Markowitz (1952).

Ressalta-se que, como demonstraram Rogers e Securato (2009), existem alternativas de modelos de precificação de ativos em relação ao CAPM, como o proposto por Fama e French (1993) e o Beta Reward Approach. O primeiro é uma alternativa mais completa ao CAPM de Sharpe (1964) e Lintner (1965) por adicionar fatores 
relacionados ao tamanho das empresas e da relação do valor de mercado com o patrimônio investido. O segundo modelo supõe que o beta deve significar a medida de risco que os investidores assumem, sendo estimado na forma de risco médio.

Porém, o resultado da comparação dos três modelos em Rogers e Securato (2009) mostrou que, para o Brasil, há maior apoio a modelos que utilizem o beta do $C A P M$ ou a modelos que utilizem uma combinação do beta com um fator que capte a diferença de tamanho das empresas. Esse segundo fator serviria como uma ferramenta auxiliar, pois o beta do CAPM seria significativo com ou sem o auxílio desse fator.

As primeiras etapas deste artigo já foram tratadas na literatura nacional, e o resultado, em geral, é que os distintos níveis de governança geram resultados que sugerem menor risco para ativos com maior transparência, como observado neste artigo. Por outro lado, poucos estudos comparam o desempenho dos índices de governança propostos pela Bovespa. Assim, na terceira etapa deste estudo foi formada uma carteira de variância mínima que considerou todas as ações classificadas em diferentes níveis de governança corporativa, além de utilizar a carteira já formada para os ativos de Novo Mercado. As duas carteiras são utilizadas para comparações com as carteiras de governança corporativa propostas pela BM\&FBovespa. Essa comparação é feita pelo desempenho e a partir da estimação do beta de mercado obtido com base no modelo CAPM.

O presente artigo é composto por quatro seções, incluindo esta introdução. Na seção 2 apresenta-se uma breve revisão bibliográfica acerca do tema. Na seção 3, são discutidos os procedimentos metodológicos utilizados neste trabalho. Na seção 4 expõem-se os resultados encontrados. Por fim, são tecidas as considerações finais.

\section{ESTUDOS APLICADOS À ANÁLISE DA GOVERNANÇA CORPORATIVA}

A expansão das discussões, pesquisas e incorporações dos métodos de governança corporativa no Brasil acaba por acumular uma base teórica considerável sobre o tema, que, ao gerar novas descobertas e mensurar os resultados, denota a eficiência na redução do risco por meio das melhorias na comunicação com os envolvidos nas operações da firma, além das melhorias na qualidade das demonstrações financeiras.

Há vários estudos aplicados à economia brasileira e que procuram tratar os benefícios da adoção dos princípios da governança corporativa. A discussão proposta por Okimura (2003) é um desses trabalhos que foi direcionado para a verificação da influência das estruturas de propriedade e controle sobre o valor de mercado e desem- 
penho das empresas brasileiras. Esse autor observou que a concentração de capital nas mãos de um único acionista apresenta relação negativa à geração de valor para a empresa, enquanto a concentração de votos em um único acionista apresenta influência positiva à geração de valor para a empresa. Sobre a influência no desempenho das empresas, pela concentração de capital ou de votos em um único acionista, não foram encontrados resultados significativos.

Já Silveira (2004), ao investigar o conjunto de mecanismos pelos quais os investidores garantem que obterão o retorno sobre suas aplicações, acabou por encontrar evidências no Brasil de que o nível de governança alcançado pelas empresas apresenta forte relação com o valor de mercado destas, explicando que as empresas que possuem um corpo gestor distinto são as mais valorizadas, embora não se possa comprovar seu desempenho superior.

Para meios conceituais, Borges e Serrão (2005, p. 112) destacam:

\begin{abstract}
A governança corporativa está relacionada à gestão de uma organização, sua relação com os acionistas (shareholders) e demais partes interessadas (stakeholders): clientes, funcionários, fornecedores, comunidade, entre outros. Nos países anglo-saxões, sua essência está baseada em mecanismos de solução para o conflito de agência, decorrente da assimetria informacional e conflito de interesses entre as partes envolvidas (proprietários e administradores).
\end{abstract}

Nesse sentido, Malacrida e Yamamoto (2006) ressaltam que são incorporados os métodos de governança aos principais conceitos do campo da Contabilidade, como: transparência (disclosure), equidade, prestação de contas (accountability) e devida responsabilidade corporativa (sustentabilidade) para explicar e solucionar os conflitos de interesse existentes.

Essa associação fez com que surgisse uma série de estudos empíricos. Um desses foi o desenvolvido por Terra e Lima (2006), em que eles investigaram se a divulgação das informações financeiras das empresas brasileiras de níveis diferenciados de governança corporativa causa o mesmo efeito sobre o mercado, quando comparadas a empresas que não estavam classificadas nos níveis de governança. Foi observado que as empresas das subamostras estatais e com atraso na divulgação dos demonstrativos financeiros ficaram em situação de desvantagem financeira em relação às empresas das subamostras privadas e sem atraso nas demonstrações financeiras. Estas últimas apresentaram retornos positivos e significativos no dia da divulgação dos dados contábeis. 
No entanto, ao se afastar das avaliações estruturais de empresas brasileiras e considerar apenas as preferências do consumidor racional, que preconiza a avaliação de investimentos por meio da regra risco-retorno, é possível observar outra forma de influência dos métodos de governança corporativa.

Entre esses, evidencia-se o estudo proposto por Macedo, Mello e Tavares Filho (2006), que procuraram analisar se a adesão ao Nível 1 de governança alterou o risco dos investimentos direcionados, exclusivamente, às companhias classificadas nesse nível de governança. Os autores utilizaram o Teste-t e o teste de Wilcoxon para duas amostras emparelhadas com o método “antes e depois” da adesão das companhias ao nível de governança já mencionado. A conclusão do estudo foi que não houve alteração significativa no risco sistemático dos ativos após a adoção de medidas de maior qualidade para as informações prestadas. O estudo considerou o período de 24 meses anterior à alteração e o período de 24 meses após a alteração, significando que mudanças nos betas (ou risco sistemático) não aconteceram durante todo o período.

Posteriormente, Malacrida e Yamamoto (2006) enfatizaram que investir em ações envolve a aceitação de determinado grau de risco, determinado pela volatilidade dos retornos dos ativos. Dessa maneira, ativos com retornos esperados mais centrados na média em determinado período - com menor desvio padrão - possuem maiores chances de apresentar uma melhor aproximação da performance esperada para o futuro, sugerindo que esses ativos de menor volatilidade devem ser adquiridos por investidores que buscam reduzir o risco nas suas operações.

Essa conclusão foi obtida a partir de um conjunto de testes estatísticos (análises de Cluster e variância e testes de Kolmogorov-Smirnov, Homogeneidade de Variâncias de Levene, Tukey HSD e Kruskal-Wallis) que consideraram três grupos de ações distintas, classificadas de acordo com o nível de governança corporativa dos ativos. A ideia era encontrar evidências factíveis sobre a hipótese de que a volatilidade decresce com o aumento da transparência das empresas. De modo geral, os resultados obtidos mostraram que há diferenças de volatilidade entre os três grupos e houve diferença significativa entre o grupo de menor e o de maior conjunto de informações prestadas, resultando em uma redução significativa na volatilidade.

As conclusões de Monte et al. (2010) corroboram a hipótese que há diferença no risco entre os diferentes níveis de governança. Nesse caso, os autores verificaram a presença de betas diferentes, em carteiras de variância mínima de diferentes níveis de governança, com maior risco para aquelas carteiras que se mostraram menos transparentes. Essa pesquisa também confrontou os resultados obtidos a partir do modelo CAPM com advindos dos modelos de volatilidade generalizados (GARCH) e, mais 
uma vez, verificou que há uma relação inversa entre o risco e o número de informações prestadas pelas empresas.

Esses argumentos foram utilizados como motivação para o artigo desenvolvido por Besarria et al. (2015, p. 18), em que, segundo eles, a redução do risco também gera melhor precificação das ações, o que, por sua vez, incentiva novas aberturas de capital e novas emissões, fortalecendo o mercado acionário como alternativa de financiamento às empresas.

Além disso, Besarria et al. (2015) também encontraram resultados que corroboram a hipótese que o risco é influenciado pelo grau de transparência das corporações. Apesar de não considerar alterações no nível de classificação das ações, uma das conclusões desse trabalho foi a evidenciação de betas reduzidos conforme se aumente o nível de governança corporativa, resultado esse obtido por meio da utilização do CAPM condicional.

Os trabalhos de Monte et al. (2010) e Besarria et al. (2015) também são dedicados à avaliação de persistência de volatilidade nas carteiras de variância mínima. Eles assumem que a reação a um choque negativo de mercado se dissipa mais rapidamente caso a persistência à volatilidade seja reduzida. Os resultados que obtiveram confirmam a influência positiva dos métodos de governança na redução da persistência, pelo fato de as carteiras de maior transparência reduzirem significativamente a influência de choques de mercado ao longo do tempo.

Além dos estudos aplicados à economia brasileira, há alguns trabalhos internacionais que também se propõem a explicar a relação entre governança corporativa e desempenho dos preços dos ativos, dentre os quais se destacam os de: Kleffner, Lee e McGannon (2003), Ferreira e Laux (2007), Lambert, Leuz e Verrecchia (2007), Owala (2010), Acharya et al. (2011), Brezeanu (2011), entre outros.

Na discussão proposta por Kleffner, Lee e McGannon (2003) o propósito foi identificar os fatores que influenciam o uso de técnicas de gerenciamento de risco por empresas do Canadá. Os resultados mostraram que a decisão de adesão ao uso dessas técnicas era principalmente influenciada por profissionais consultores e executivos das empresas e por resultados e diretrizes de empresas participantes da Bolsa de Valores de Toronto. As empresas que utilizaram os princípios de governança verificaram que o melhor desempenho financeiro de setores específicos das corporações estava diretamente ligado ao uso dos princípios de governança.

Já Owala (2010) busca evidências que corroborem a adoção de estratégias de governança. Sua análise foi realizada a partir da correlação entre os níveis de governança das empresas e o nível de retorno dos ativos, utilizando uma amostra das firmas 
do S\&P 500 de 2008. Os achados mostraram que não há diferenças significativas nos retornos entre as firmas de alto e baixo nível de governança, verificando, ainda, a impossibilidade de prever retornos futuros pela qualidade de governança corporativa existente.

Por outro lado, Brezeanu et al. (2011) investigaram a importância da governança corporativa por meio de variáveis como rentabilidade e alavancagem, em uma série de dados de 100 empresas listadas na NASDAQ, entre os anos 1997 e 2007. Como estratégias de gerenciamento de risco são relacionadas à estrutura corporativa, as estratégias são orquestradas de acordo com a capacidade de atrair investimentos. Portanto, foi observado que as firmas mais líquidas possuem essa capacidade; além disso, firmas maiores possuem maior capacidade de gerar lucro e tangibilidade. Por fim, eles ressaltam que grandes alianças de mercado são bem- vistas pelo mercado em razão da propensão de gerar alavancagem.

Há uma série de outros estudos que tratam da importância da governança corporativa no combate a crimes contábeis, comportamento oportuno de gerentes, papel da mídia na governança, governança nas empresas públicas, entre outros. Essas temáticas estão presentes em: Agrawal e Cooper (2017), Ararat, Black e Yurtoglu (2017), Schmidt e Fahlenbrach (2017), Shi, Brian e Hoskisson (2017), Liu, McConnell e Xu (2017).

Esta seção foi importante para mostrar que a temática governança corporativa associada à redução de risco já foi bastante debatida na literatura e que os resultados, de modo geral, reforçam o argumento de que maior transparência influencia negativamente o risco do investimento. Por outro lado, os estudos não propõem carteiras alternativas de governança não confrontam o desempenho das carteiras de governança corporativa propostas pela BM\&FBovespa com métodos alternativos de diversificação, como é proposto neste artigo.

\section{PROCESSOS METODOLÓGICOS}

O procedimento metodológico adotado segue os métodos usuais de construção do banco de dados e estimação do modelo CAPM para carteiras de ativos. A experiência brasileira remete a trabalhos recentes, como os de Sucolotti (2007), Rogers e Securato (2009), Bodur (2011) e Besarria et al. (2015), que serviram como ferramentas direcionais para este trabalho. 


\subsection{DADOS}

Os dados utilizados são referentes aos preços de fechamento diário de 01 de junho de 2012 a 04 de julho de 2016 das empresas da BM\&FBovespa, retirados do banco de dados da empresa de consultoria Economática. O motivo para a utilização de tal período está ligado à disponibilidade de dados de ativos de Nível 2, visto que, caso fosse ampliado o período, haveria restrição à quantidade de ativos nessa carteira. No período selecionado estiveram disponíveis as cotações de 37 ativos no Nível 1, 16 ativos no Nível 2 e 93 ativos no Novo Mercado.

Foi também utilizada a série histórica do ativo livre de risco, representada pelo Certificado de Depósito Interbancário (CDI), retirada do site da Central de Custódia e Liquidação de Títulos Privados (CETIP), além das séries históricas retiradas do site da BM\&FBovespa, que são: índice de mercado (IBovespa), índice de mercado para governança corporativa (IGC) e índice de mercado para ativos de Novo Mercado (IGC-NM).

\subsection{CONSTRUÇÃO DAS CARTEIRAS, ÍNDICE SHARPE E CAPM}

A construção das carteiras correspondentes aos três níveis de governança corporativa segue o método amplamente aceito de Markowitz (1952), que utiliza a diversificação como ferramenta de redução do risco de um portfólio, tornando mais eficiente seu desempenho.

O retorno diário das ações - única informação necessária para avaliar os ativos pelo binômio risco-retorno e construir as carteiras - é obtido pelo método de retorno simples, como segue:

$$
R_{i, t}=\left[\left(P_{i, t} / P_{i, t-1}\right)-1\right] * 100
$$

em que $R_{i, t}$ é o retorno da ação i no dia t; $P_{i, t}$ é a cotação de fechamento da ação i no dia t e $P_{i, t-1}$ representa a cotação de fechamento da ação i no dia anterior ao dia t. Já o retorno das carteiras é obtido pela seguinte fórmula:

$$
E\left(R_{p}\right)=\sum_{i=1}^{n} R_{i} X_{1}
$$


Sendo $E\left(R_{p}\right)$ o retorno esperado da carteira. $R_{i}$ é o retorno esperado do ativo i e $X_{i}$ é a participação do ativo i na carteira. Além desses, o risco da carteira é obtido por:

$$
\sigma_{p}=\left[\Sigma_{i=1}^{n} \Sigma_{j=1}^{n} X_{i} X_{j} \rho_{i j} \sigma_{i} \sigma_{j}\right]^{\frac{1}{2}}
$$

caracterizando $\sigma_{p}$ como o risco da carteira. $X_{i}$ e $X_{j}$ representam a participação dos ativos $i$ e $j$ na carteira, $\rho_{i j}$ é o coeficiente de correlação entre os ativos e $\sigma_{i}$ e $\sigma_{j}$ é o desvio padrão dos ativos $i$ e $j$.

A otimização das carteiras é obtida pela utilização da ferramenta Solver do Excel, tomando como objetivo obter o menor desvio padrão, com as seguintes restrições: ausência de alavancagem (somatório das participações igual a 100\%), ausência de vendas a descoberto (participações maiores ou iguais a zero) e taxa de rendimento médio ao dia que seja capaz de superar o rendimento total do CDI no período.

Já os índices de Sharpe dos ativos são construídos a partir da seguinte expressão:

$$
I S_{P}=\frac{E\left(R_{P}\right)-E\left(R_{f}\right)}{\sigma_{P}}
$$

$I S_{F}$ em que é o índice de Sharpe do portfólio $P, E\left(R_{P}\right)$ o retorno esperado pelo portfólio $P, E\left(R_{f}\right)$ seria o retorno médio do ativo livre de risco e $\sigma_{P}$ é o desvio padrão do portfólio $P$. Por fim, ressalta-se que o modelo CAPM pode ser expresso por:

$$
R_{p, t}-R_{f, t}=\alpha_{p}+\beta_{p}\left(R m_{t}-R f_{t}\right)+e_{p, t}
$$

em que $R_{p, t}-R_{f, t}$ é o prêmio pelo ativo $i$ em relação ao CDI ou ativo livre de risco no dia $t, R m_{t}-R f_{t}$ caracteriza o excesso de retorno de mercado em relação ao CDI no dia $t, \alpha_{P}$ indica o retorno do ativo $P$, independente da influência do fator mercado, $\beta_{P}$ é o coeficiente de sensibilidade do ativo $P$ ao fator mercado e $e_{P, t}$ é o resíduo do modelo referente ao ativo $P$ no dia $t$. 


\subsection{ESTIMATIVA DOS BETAS VARIANDO NO TEMPO}

A discussão sobre os betas variando no tempo segue os procedimentos utilizados por Besarria et al. (2015). Os autores partem da versão condicional do modelo CAPM, proposto por Bollerslev, Engle e Wooldridge (1988). Esses autores assumem $y_{t}$ como um vetor de retornos excedentes, representado pela diferença entre o retorno nominal dos ativos e o ativo livre de risco durante o período $t$, e assumem $\mu_{t}$ e $H_{t}$ como o vetor da média condicional e a matriz covariância condicional desses retornos dada a partir do conjunto de informações disponíveis no momento $t-1$, assumindo $\omega_{t-1}$ ser o vetor de pesos do período $t-1$ de modo que o excesso de retorno do mercado é definido como $y_{M t}=y_{t}^{\prime} \omega_{t-1}$. Em seguida, o vetor de covariâncias do mercado é $H_{t} \omega_{t-1}$, e o $C A P M$ requer

$$
\mu_{t}=\delta H_{t} \omega_{t-1}
$$

em que $\delta$ é uma constante de proporcionalidade, derivada por Jensen (1972), e representa uma medida agregada de aversão ao risco relativo.

A variância condicional do excesso de retorno do mercado é $\sigma_{M_{t}}^{2}=\omega_{t-1}^{\prime} H_{t} \omega_{t-1}$, e a média condicional é $\mu_{M_{t}}=\omega_{t-1} \mu_{t}$, que a partir de (6) pode ser escrita como

$$
\mu_{M_{t}}=\delta \sigma_{M_{t}}^{2}
$$

mostrando que $\delta$ expressa o trade-off entre a média e a variância de mercado. Definindo o beta do ativo como a razão entre a covariância do ativo com o mercado e a variância da carteira de mercado, $\beta_{t}=H_{t} \omega_{t-1} / \sigma_{M_{t}}^{2}$ e substituindo (6) em (7), obtém-se

$$
\mu_{t}=\beta_{t} \mu_{M_{t}}
$$

Essa expressão representa o modelo CAPM em termos dos momentos condicionais e reflete as informações disponíveis aos agentes no momento da tomada de 
decisão sobre a formação do portfólio. Destaca-se que, como a matriz de covariância dos retornos varia ao longo do tempo, os retornos médios e os betas também são variáveis com o tempo.

\section{RESULTADOS}

A discussão inicial desta seção é destinada à avaliação das carteiras desenvolvidas para cada nível de governança, sendo analisado o desvio padrão, o rendimento diário e o Índice de Sharpe dos ativos, o que está em acordo com as análises usuais do mercado financeiro e testes empíricos na área. Como já ressaltado foi adotado como ativo livre de risco o CDI, sendo esse o parâmetro-base para postular avaliações aos demais ativos de mercado. Sua rentabilidade é percebida como o limite inferior a ser exigido em investimentos que envolvem risco; dessa maneira, o investidor recebe um prêmio ou excedente por se expor ao risco.

Assim, a estratégia adotada para otimizar o risco das carteiras foi de minimização do desvio padrão a partir da ferramenta Solver do Excel. Em outras palavras, foi definido um nível de rendimento aceitável (maior ou igual ao do ativo livre de risco) com a menor trajetória de volatilidade possível no período. Como pode ser observado na Tabela 1, os três portfólios obtiveram retorno esperado superior ao CDI, porém com risco em grandezas diferentes. Além disso, verifica-se que o índice de mercado, representado pelo Ibovespa, apresentou rentabilidade diária média abaixo do CDI.

Tabela 1 - Resumo dos ativos e participação dos ativos nas respectivas carteiras, no período de 01 de junho de 2012 a 04 de julho de 2016 (carteiras N1, N2 e NM, além do CDI e o IBOV)

\begin{tabular}{lrrrrr}
\hline & $\mathbf{N 1}$ & $\mathbf{N 2}$ & $\mathbf{N M}$ & $\mathbf{C D I}$ & IBOV \\
Desvio padrão & $1,05 \%$ & $0,95 \%$ & $0,73 \%$ & $0,01 \%$ & $1,51 \%$ \\
Rendimento diário & $0,046 \%$ & $0,046 \%$ & $0,045 \%$ & $0,040 \%$ & $0,010 \%$ \\
Índice de Sharpe & $0,57 \%$ & $0,63 \%$ & $0,68 \%$ & $0,00 \%$ & $-1,98 \%$ \\
Ativos disponíveis & 37 & 16 & 93 & - & - \\
Ativos selecionados & 12 & 7 & 38 & - & - \\
\hline
\end{tabular}

Fonte: os autores.

A partir dos dados da Tabela 1 também é possível verificar que as carteiras N1, N2 e NM obtiveram desempenho melhor que o Ibovespa. É importante destacar que o período tratado neste estudo considera momentos de grande instabilidade econômica local e mundial, marcados pela crise do subprime, período eleitoral e episódios de corrupção ligados às principais empresas brasileiras. A junção desses fatores 
fortalece a hipótese da diversificação de investimentos e busca a redução de risco, por meio de investimentos com maior grau de transparência e governança corporativa. Dessa forma, os resultados apresentados na Tabela 1 ilustram, mesmo que de forma embrionária, essas características, sendo esperado que quanto mais alto o nível de informações prestadas aos investidores, melhor seja a avaliação risco-retorno e a atratividade do ativo.

Essa primeira análise foi útil para mostrar que houve uma sensível melhora no desempenho da carteira conforme aumentou o nível de informações prestadas, com o nível de risco no portfólio NM sendo o mais baixo no período e, apesar da proximidade nos valores, a carteira N2 apresentou menor risco que a N1. Essa afirmação sobre o desempenho das carteiras é melhor condensada e exposta ao se analisar o índice de Sharpe, que relaciona o retorno esperado excedente dos ativos diante do CDI com o risco observado no período do próprio ativo.

Ao observar os resultados expostos na Tabela 1 é possível verificar que, de fato, a carteira NM apresentou o índice de Sharpe com melhor desempenho, apresentando um valor de $0,68 \%$. Intuitivamente, para cada $1 \%$ de risco adicionado na carteira ela apresentará um acréscimo de rendimento, em média, de 0,68\%. Na sequência verificou-se que a carteira N2 apresentou um valor de 0,63\% e, com pior desempenho, tem-se a carteira N1, com um índice de Sharpe médio de 0,57\%. Em relação à carteira de mercado, percebe-se que as ações mais negociadas do mercado no período apresentaram um baixo desempenho, fazendo com que o índice de Sharpe dessa carteira fosse de $-1,98 \%$.

\subsection{RESULTADO DAS ESTIMATIVAS DOS BETAS DE MERCADO}

O Gráfico 1 exibe a dispersão entre os retornos excedentes das três carteiras de governança corporativa e o retorno excedente do índice de mercado. Apesar de ser uma análise simplista e frágil, é possível extrair os indícios de uma associação linear positiva entre o Ibovespa e as três carteiras tratadas, além de observar um comportamento que também sugere maior dispersão na carteira N1, que apresentou maior desvio padrão diante de outras carteiras. 
Gráfico 1 - Relação entre os retornos excedentes das carteiras com níveis diferenciados de governança corporativa e o excesso de retorno do índice de mercado
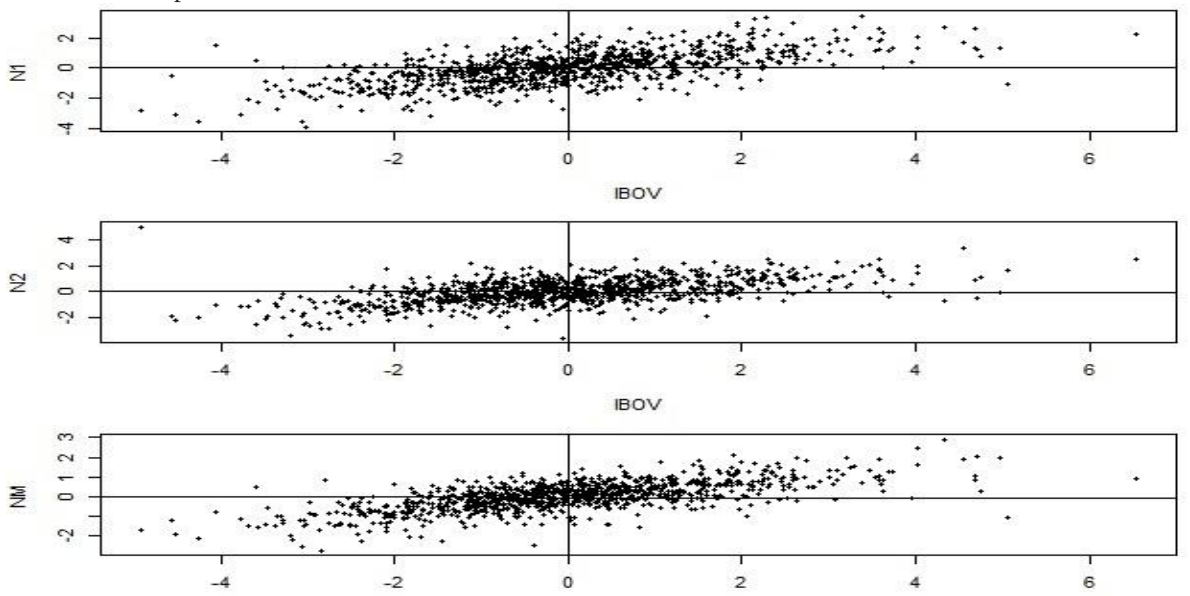

Fonte: os autores.

No entanto, uma maneira mais formal de se tratar essa relação é por meio do modelo CAPM. Com o CAPM é possível medir o nível de influência do desempenho do mercado no retorno dos ativos, estabelecendo a carteira de mercado como a única variável explicativa do rendimento do ativo ou portfólio. As estimativas do CAPM, obtidas a partir do método dos mínimos quadrados ordinários, estão presentes na Tabela 2.

Tabela 2 - Estimativa dos parâmetros pelo CAPM para as carteiras N1, N2 e NM

\begin{tabular}{lrrr}
\hline & $\mathbf{N 1}$ & $\mathbf{N 2}$ & $\mathbf{N M}$ \\
\hline Intercepto & 0,0002 & 0,0002 & 0,0002 \\
t-valor & 0,74 & 0,65 & 0,93 \\
Beta de mercado & 0,439 & 0,344 & 0,326 \\
t-valor & 25,9 & 20,8 & 29,2 \\
Coef. de determinação & $40,08 \%$ & $30,12 \%$ & $45,86 \%$ \\
Teste Durbin-Watson & & & \\
DW & 2,0327 & 2,2841 & 2,0283 \\
\hline p-valor & 0,698 & 1 & 0,67 \\
\hline
\end{tabular}

Fonte: os autores.

Direcionar, inicialmente, a discussão para a análise econométrica é afirmar que, por meio do teste $t$ de Student, os betas de mercado apresentaram significância estatística ao nível de confiança de 95\%. Já os interceptos não foram significativos estatisticamente, indicando a ausência de vendas a descoberto na estimativa de risco sistemático das carteiras. 
De forma complementar, foram tratados os coeficientes de determinação, os quais indicaram grau de ajuste aceitável, de grandeza semelhante aos obtidos por Rogers e Securato (2009) e Bodur (2011). Já o teste de Durbin-Watson sugeriu a aceitação da hipótese nula de ausência de autocorrelação nos resíduos para as três carteiras; dessa forma, há conformidade com a hipótese de independência dos resíduos.

Após a discussão estatística das estimativas é possível afirmar que os resultados obtidos nessa etapa reforçam a hipótese que há diminuição no nível de risco sistemático presente nas carteiras, conforme aumenta o nível de governança corporativa nos portfólios. Percebe-se, por exemplo, que o coeficiente de 0,439 da carteira $\mathrm{N} 1$ indica que esta apresentou risco e retorno superior à carteira mais transparente, representada pela NM.

Esse achado está em conformidade com os obtidos por Besarria et al. (2015), que verificaram, por meio das estimativas das volatilidades, que as ações das empresas do Novo Mercado são as que apresentam menor risco, seguidas pelas ações classificadas no Nível 2 e Nível 1, respectivamente.

\subsection{RESULTADOS PARA COMPARAÇÕES COM ÍNDICES DE GOVERNANÇA CORPORATIVA}

O exercício desenvolvido anteriormente é repetido nesta seção com o propósito de comparar o desempenho das carteiras de mercado propostas pela BM\&FBovespa, utilizadas para representar as corporações classificadas com diferentes níveis de governança corporativa, com carteiras de governança que foram criadas a partir do princípio da minimização de risco proposto por Markowitz (1952).

Inicialmente foi criada uma carteira geral que inclui todos os níveis de governança, também conhecida como índice ou carteira de governança geral (CGOV). Parte-se do princípio de que investidores incluem em seu portfólio os ativos com menor nível de risco, como postula a teoria de minimização de risco, e não apenas os que apresentam o maior liquidez, como propõe a BM\&FBovespa.

A criação de um portfólio que inclua todos níveis de governança apresenta a vantagem de possibilitar melhor desempenho, em decorrência da maior possibilidade de diversificação e quantidade de informações. Partindo desse conjunto de dados é criada a carteira de governança corporativa geral, como apresentada na Tabela $3 .^{22}$

\footnotetext{
${ }^{2}$ Ressalta-se que as matrizes de variância-covariância podem ser solicitadas aos autores, caso haja interesse.
} 
A partir da Tabela 3 é possível verificar que, aproximadamente, 57\% dos ativos da carteira CGOV são provenientes da categoria NM, e o restante é distribuído entre N1 e N2. O desempenho dessa carteira foi comparado ao apresentado pelo Índice de Ações de Governança Corporativa Diferenciada (IGC), proposto pela BM\&FBovespa. Esse índice foi desenvolvido com o objetivo de representar o desempenho médio das ações listadas entre os três níveis de governança, em que ele é criado na forma de uma carteira diversificada representada por um índice de retorno total e que considera a liquidez e o número de informações prestadas.

Tabela 3 - Resultado da diversificação da carteira de governança geral, que inclui ativos dos três níveis de governança corporativa

\begin{tabular}{lrrrr}
\hline & N1 & N2 & NM & CGOV \\
\hline $\begin{array}{l}\text { Ativos disponíveis } \\
\text { Ativos selecio- } \\
\text { nados }\end{array}$ & 37 & 16 & 93 & 146 \\
$\begin{array}{l}\text { Participação } \\
\text { Média }\end{array}$ & 18 & 9 & 35 & 62 \\
Mínima & $0,77 \%$ & $2,27 \%$ & $1,88 \%$ & $1,61 \%$ \\
Máxima & $0,00 \% *$ & $0,00 \% *$ & $0,00 \% *$ & $0,00 \% *$ \\
Fonte: 0 * autores. & $4,62 \%$ & $9,37 \%$ & $10,41 \%$ & $10,41 \%$ \\
\hline
\end{tabular}

Fonte: os autores.

Nota: * Valores positivos e próximos a zero.

Além disso, foi comparado o desempenho da carteira de Novo Mercado, já apresentada anteriormente, com o Índice de Governança Corporativa - Novo Mercado (IGC-NM), que também é formulado pela BM\&Fbovespa, possuindo metodologia de construção similar ao IGC. Segundo a BM\&Fbovespa (METODOLOGIA..., 2015b), a única diferença para a construção do IGC-NM em relação ao IGC é que a ponderação dos ativos não depende do impacto de fatores de diferenciação, pelo fato de não haver diferentes níveis de governança corporativa em uma carteira. Dessa forma, todos os ativos na carteira IGC-NM possuem ponderação dependente apenas do valor de mercado em free-float, não sendo infladas por nenhum fator de diferenciação.

Como pode ser observado na Tabela 4, tanto o IGC quanto o IGC-NM apresentaram, no período tratado, um desempenho inferior ao rendimento do ativo livre de risco, CDI, em que este último teve um rendimento médio de $0,040 \%$ ao dia. 
Tabela 4 - Desempenho das carteiras CGOV, IGC, NM e IGC-NM

\begin{tabular}{lrrrr}
\hline & CGOV (\%) & IGC (\%) & NM (\%) & IGC-NM (\%) \\
\hline Desvio padrão & 0,62 & 1,19 & 0,73 & 1,10 \\
Rendimento diário & 0,043 & 0,026 & 0,045 & 0,035 \\
Índice de Sharpe & 0,48 & $-1,17$ & 0,68 & $-0,48$ \\
\hline
\end{tabular}

Fonte: os autores.

Por outro lado, conforme exposto na Tabela 4, verifica-se que as carteiras CGOV e NM apresentaram risco inferior ao IGC e ao IGC-NM e retorno acima do CDI. Outro dado comparativo importante, observado no Gráfico 2, é a análise da fronteira eficiente para as carteiras descritas anteriormente.

A fronteira eficiente apresenta o conjunto de combinações de ativos possíveis, em que o peso ou participação de cada um influencia no desempenho da carteira. A análise da fronteira eficiente considera as composições que, para cada patamar de risco, apresentam o maior nível de retorno possível.

Dessa forma, as características das fronteiras eficientes das carteiras CGOV e NM, representadas no Gráfico 2, indicam que para o nível de risco e retorno apresentado pelas carteiras IGC e IGC-NM, esses portfólios são preferíveis. No caso da CGOV, por exemplo, para o mesmo nível de risco apresentado pela IGC (1,20\%) essa carteira apresentou um retorno médio de aproximadamente $0,14 \%$. A mesma característica é observada na comparação entre as carteiras NM e IGC-NM, com a primeira apresentando desempenho preferível em relação à segunda.

Gráfico 2 - Fronteira eficiente das carteiras CGOV, IGC, NM e IGC-NM
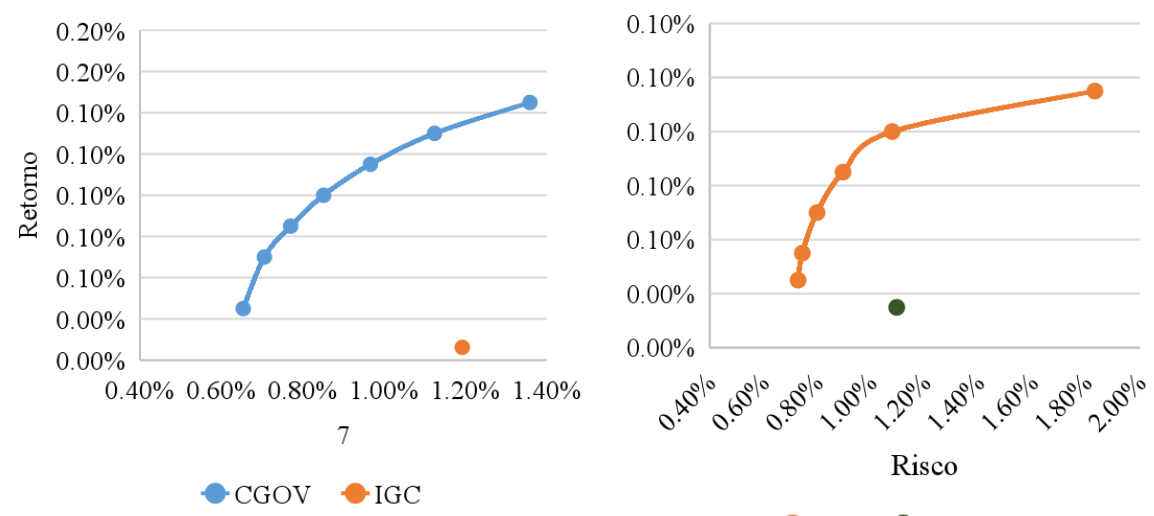

Risco

Fonte: os autores. 
Dada a diferença de desempenho entre essas carteiras, é importante ressaltar que a origem dessa disparidade é proveniente das diferenças nos métodos de formação das carteiras. No caso tratado neste artigo, é adotado o princípio da Teoria do Portfólio de Markowitz, mas esse não é o mesmo método empregado pelos índices da BM\&FBovespa, como já destacado. Para o IGC, por exemplo, o objetivo não é a redução do risco por diversificação, mas indicar o retorno médio dos ativos mais líquidos dos três níveis de governança corporativa. Porém, há indícios que os princípios propostos por Markowitz (1952) são preferíveis aos adotados pela BM\&FBovespa.

A discussão subsequente apresenta as estimativas dos betas de mercado, obtidos a partir do método de mínimos quadrados ordinários, e que expressam a associação entre os excessos de retorno das carteiras CGOV, IGC, NM e IGC-NM com o índice de mercado, como pode ser visto na Tabela 5.

A partir da Tabela 5 é possível verificar que as carteiras CGOV e NM apresentaram os resultados esperados, obtendo risco sistemático inferior às IGC e IGC-NM. Ressalta-se que o intercepto dessas regressões não apresentou significância estatística ao nível de confiança de 95\%, indicando ausência de vendas a descoberto. Por outro lado, os valores obtidos para os betas de mercado, em todos os modelos tratados, foram significativos estatisticamente, e as carteiras CGOV e NM apresentaram risco inferior às carteiras IGC e IGC-NM. Essa conclusão pode ser extraída a partir dos valores obtidos para as estimativas dos betas das carteiras, e, como pode ser visto na Tabela 5, as carteiras CGOV e NM apresentaram estimativas iguais a 0,269 e 0,326, sendo muito inferiores aos valores 0,759 e 0,659 obtidos, respectivamente, pelas carteiras IGC e IGC-NM.

Tabela 5 - Estimativa do CAPM para as carteiras CGOV, IGC, NM e IGC-NM

\begin{tabular}{lrrrr}
\hline & CGOV & IGC & NM & IGC-NM \\
\hline Intercepto & 0,0001 & 0,0001 & 0,0002 & 0,0001 \\
t-valor & 0,72 & 0,90 & 0,93 & 1,04 \\
Beta de mercado & 0,269 & 0,759 & 0,326 & 0,659 \\
t-valor & & & 29,2 & 70,7 \\
27,2 & & & $83,24 \%$ \\
114,67 & $42,42 \%$ & $92,89 \%$ & $45,86 \%$ & \\
Coef. de determinação & & & & 1,8561 \\
Teste Durbin-Watson & & & & 0,01 \\
DW & 2,0379 & 1,9045 & 2,0283 & 0,67 \\
p-valor & 0,726 & 0,064 & &
\end{tabular}


Dessa análise surge uma nova indagação: o valor estático do beta de mercado é um bom indicador para analisar o desempenho das carteiras? Ou será que há momentos nos quais as carteiras propostas pela BM\&FBovespa passam a ter um desempenho superior às carteiras CGOV e NM? As respostas para essas indagações podem ser obtidas a partir da análise do comportamento dos betas ao longo do tempo, como expresso no Gráfico 3.

Gráfico 3 - Betas condicionais das carteiras CGOV, IGC, NM e IGC-NM

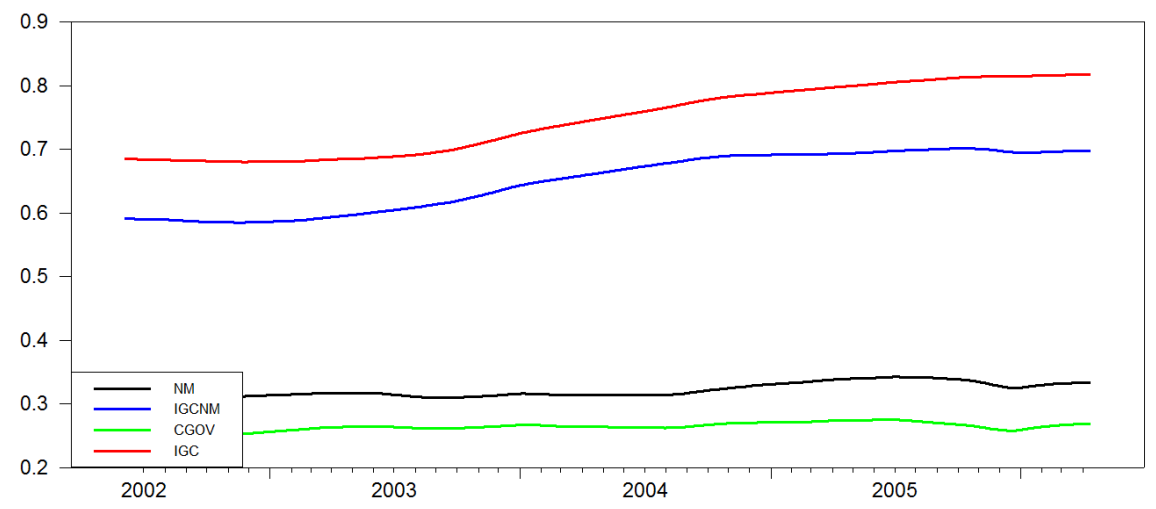

Fonte: os autores.

O Gráfico 3 corrobora as análises já apresentadas (betas estáticos) e mostra que as carteiras propostas pela BM\&FBovespa apresentaram retorno e risco superior ao risco das carteiras propostas neste artigo. Percebe-se, por exemplo, que a carteira IGC apresentou beta superior ao longo do tempo perante as demais carteiras, por outro lado, a CGOV foi a carteira que teve o menor beta condicional de mercado, seguida pelo portfólio NM.

\section{CONCLUSÃO}

Os princípios básicos da governança corporativa estão ligados ao fato de as empresas com maior transparência de mercado obterem uma melhora no processo de valorização de suas ações e uma redução no risco dos seus ativos. Com base nesse argumento, neste artigo procurou-se analisar a influência desse mecanismo de classificação das ações, conforme seu grau de transparência, na redução do risco, por meio da teoria do portfólio e do modelo CAPM. 
De modo geral, os resultados são favoráveis à hipótese de maior grau de transparência influenciando negativamente o risco. Essa mesma conclusão foi obtida em diferentes processos de análise (teoria do portfólio, medidas de posições e dispersão, CAPM estático e dinâmico). Quanto às implicações deste estudo, verifica-se que o uso de técnicas de governança corporativa contribui para a redução da volatilidade dos ativos, seja por meio da análise individual, quando comparadas as carteiras N1, N2 e NM, seja a partir da análise conjunta. Dessa forma, investidores que buscam ativos menos voláteis devem considerar o grau de transparência das ações e/ou empresas no momento de formar seu portfólio.

A principal limitação deste estudo é que, no momento da formação das carteiras N1 e N2, foi verificado que há um número restrito de ações classificadas nos segmentos N2. Assim, em discussões futuras, a formação das carteiras associará ações N1 e N2 como um índice único, podendo ser classificado como carteira de pouca transparência e comparar com o desempenho do NM, verificar se as características observadas para a economia brasileira também são válidas para outros países e analisar as características da volatilidade condicional.

\section{REFERÊNCIAS}

ACHARYA, V. et al. Corporate Governance and Value Creation: evidence from Private Equity. NYU Working Paper, i. FIN-08-032, 2011.

AGRAWAL, A.; COOPER, T. Corporate governance consequences of accounting scandals: evidence from top management, CFO and auditor turnover. Quarterly Journal of Finance, v. 7, i. 1, p. 1650014, 2017.

ARARAT, M.; BLACK, B. S.; YURTOGLU, B. B. The effect of corporate governance on firm value and profitability: time-series evidence from Turkey. Emerging Markets Review, v. 30, p. 113-132, 2017.

BESARRIA, C. N. et al. A qualidade das informações prestadas pelas empresas reduz os riscos de investimento? Uma análise empírica para os diferentes níveis de governança corporativa das empresas brasileiras. RACE, Revista de Administração, Contabilidade e Economia, Joaçaba: Ed. Unoesc, v. 14, n. 1, p. 11-38, jan./abr. 2015.

BODUR, F. J. Uma comparação entre os modelos CAPM, Fama-French e Fama-French-Carhart. Porto Alegre: Ed. UFRGS, 2011. 
BOLLERSLEV, T.; ENGLE, R. F.; WOOLDRIDGE, J. M. A Capital Asset Pricing Model with Time-varying Covariances. Journal of Political Economy, v. 96, i. 1, Feb. 1988.

BORGES, L. F. X.; SERRÃO, C. F. de B. Aspectos de Governança Corporativa Moderna no Brasil. Revista do BNDES, Rio de Janeiro: GEDIT, v. 12, n. 24, p. 111148, dez. 2005.

BREZEANU, P. et al. Does Corporate Governance impact risk management system? Theoretical and Applied Economics, v. 18, i. 4, p. 49-64, 2011.

CARMONA, C. U. de M. Finanças corporativas e mercados. São Paulo: Atlas, 2009.

FAMA, E.; FRENCH, K. Common risk factors in the returns on stocks and bonds. Journal of Financial Economics, v. 33, i. 1, p. 3-56, 1993.

FERREIRA, M.; LAUX, P. Corporate Governance, Idiosyncratic Risk, and Information Flow, Journal of Finance, v. 62, p. 951-989, 2007.

JENSEN, M. C. The Foundations and Current State of Capital Market Theory. The Bell Journal of Economics and Management Science, v. 3, i. 2, 1972.

KLEFFNER, A. E.; LEE, R. B.; MCGANNON, B. The effect of Corporate Governance on the use of enterprise risk management: evidence from Canada. Risk Management and Insurance Review, v. 6, i. 1, p. 53-73, Feb. 2003.

LAMBERT, R. A.; LEUZ, C.; VERRECCHIA, R. Accounting Information Disclosure, and the Cost of Capital. Journal of Accounting Research, v. 45, p. 385-420, 2007.

LINTNER, John. The valuation of risk assets and the selection of risky investments in stock portfolios and capital budgets. Review of Economics and Statistics, v. 47, i. 1 , p. 13-37, 1965.

LIU, B.; MCCONNELL, J. J.; XU, W. The power of the pen reconsidered: The media, CEO human capital, and corporate governance. Journal of Banking \& Finance, v. 76, p. 175-188, 2017.

MALACRIDA, M. J. C.; YAMAMOTO, M. M. Governança Corporativa: nível de evidenciação das informações e sua relação com a volatilidade das ações do Ibovespa. RC\&F - Revista de Contabilidade e Finanças da USP, São Paulo, p. 65-79, 2006. Edição Comemorativa. 
MARKOWITZ, H. M. Portfolio selection. The Journal of Finance, v. 7, i. 1, p. 7791, Mar. 1952.

METODOLOGIA do índice Bovespa. BM\&FBOVESPA. São Paulo, abr. 2015. Disponível em: <http://www.bmfbovespa.com.br/lumis/portal/file/fileDownload. jsp?fileId=8A828D29514A326701516E695D7F65C0> . Acesso em: 05 mar. 2017.

METODOLOGIA do índice de ações com governança corporativa diferenciada (IGC). BM\&FBOVESPA, São Paulo, abr. 2015a. Disponível em: <http:// www.bmfbovespa.com.br/lumis/portal/file/fileDownload.jsp?fileId=8A828D29514A326701516E7E43FB1263>. Acesso em: 05 mar. 2017.

METODOLOGIA do índice de governança corporativa - Novo Mercado (IGC-NM). BM\&FBOVESPA, São Paulo, abr. 2015b. Disponível em: <http://www.bmfbovespa.com.br/lumis/portal/file/fileDownload.jsp?fileId=8A828D29514A326701516E7BF5040C00>. Acesso em: 05 mar. 2017.

MONTE, P. A. do et al. Existe relação entre Governança Corporativa e volatilidade? Um estudo a partir da formação de carteiras. Contabilidade Vista \& Revista, v. 21, n. 2, p. 15-44, 2010.

OKIMURA, R. T. Estrutura de propriedade, governança corporativa, valor e desempenho das empresas no Brasil. São Paulo: Ed. USP, 2003.

OWALA, A. C. Corporate Governance and stock returns: evidence from S\&P 500. Vaasa: University of Vaasa, 2010.

ROGERS, P.; SECURATO, J. R. Estudo comparativo no mercado brasileiro do Capital Asset Pricing Model (CAPM), Modelo 3-fatores de Fama e French e Reward Beta Approach. RAC-E, Revista de Administração Contemporânea Eletrônica, Curitiba, v. 3, n. 1, p. 159-179, jan./abr. 2009.

SÁ, G. T. de. Administração de investimentos, teoria de carteiras e gerenciamento de risco. Rio de Janeiro: Qualitymark, 1999.

SCHMIDT, C.; FAHLENBRACH, R. Do exogenous changes in passive institutional ownership affect corporate governance and firm value? Journal of Financial Economics, v. 124, i. 2, p. 285-306, 2017.

SHARPE, W. F. Capital asset prices: A theory of market equilibrium under conditions of risk. Journal of Finance, v. 19, i. 3, p. 425-442, Sept. 1964. 
SHI, W.; CONNELLY, B. L.; HOSKISSON, R. E. External corporate governance and financial fraud: cognitive evaluation theory insights on agency theory prescriptions. Strategic Management Journal, v. 38, i. 6, p. 1268-1286, 2017.

SILVA, M. B. da; CÂMARA, S. F. A governança corporativa como um diferencial de mercado das empresas listadas na BM\&FBovespa no ano de 2012. GeCont, v. 2, n. 1, p. 152-170, jan./jun. 2015.

SILVEIRA, A. di M. da. Governança corporativa e estrutura de propriedade: determinantes e relação com o desempenho das empresas no Brasil. 2004. Tese (Doutorado em Administração)-Universidade de São Paulo, São Paulo, 2004.

SUCOLOTTI, L. Avaliação de desempenho de carteiras: Markowitz x Índice Bovespa. Porto Alegre: Ed. UFRGS, 2007.

TERRA, P. R. S.; LIMA, J. B. N. de. Governança Corporativa e a reação do mercado de capitais à divulgação das informações contábeis. RC\&F - Revista de Contabilidade e Finanças da USP, São Paulo, p. 35-49, set./dez. 2006.

\section{Como citar este artigo:}

ABNT

BESARRIA, Cássio Nóbrega; SILVA, Herickson Santos. A efetividade da governança corporativa sobre o risco dos ativos da BM\&FBovespa. RACE, Revista de Administração, Contabilidade e Economia, Joaçaba: Ed. Unoesc, v. 16, n. 3, p. 933-956, set./dez. 2017. Disponível em: <http://editora.unoesc.edu.br/index.php/ race>. Acesso em: dia/mês/ano.

APA

Bessaria, C. N., \& Silva, H. S. (2017). A efetividade da governança corporativa sobre o risco dos ativos da BM\&FBovespa. RACE, Revista de Administração, Contabilidade e Economia, 16(3), 933-956. Recuperado em dia/mês/ano, de http://editora. unoesc.edu.br/index.php/race 